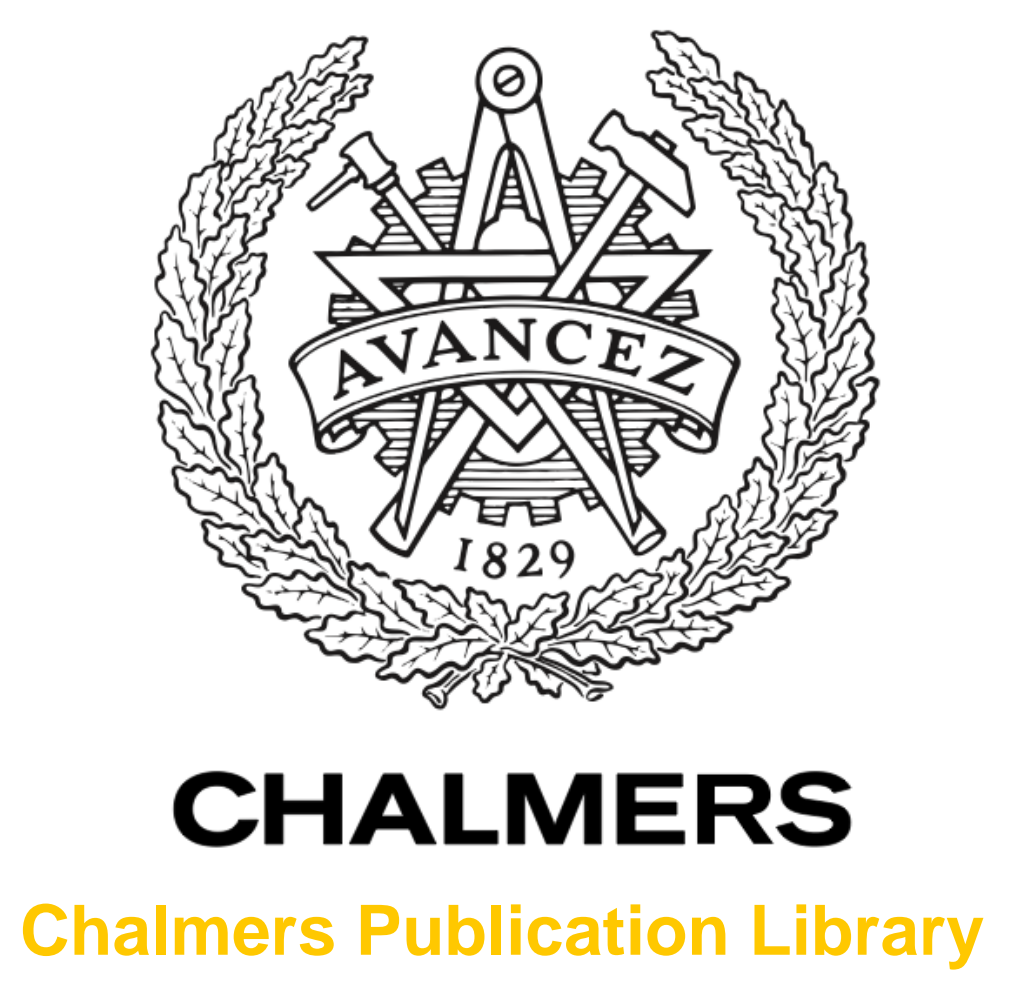

\title{
Nonlinear 3-D microwave imaging for breast-cancer screening: Log, phase and log- phase formulation
}

This document has been downloaded from Chalmers Publication Library (CPL). It is the author's version of a work that was accepted for publication in:

\section{LAPC 2011 - 2011 Loughborough Antennas and Propagation Conference}

Citation for the published paper:

Jensen, P. ; Rubaek, T. ; Mohr, J. (2011) "Nonlinear 3-D microwave imaging for breastcancer screening: Log, phase and log-phase formulation". LAPC 2011 - 2011 Loughborough Antennas and Propagation Conference

http://dx.doi.org/10.1109/LAPC.2011.6114124

Downloaded from: http://publications.lib.chalmers.se/publication/166187

Notice: Changes introduced as a result of publishing processes such as copy-editing and formatting may not be reflected in this document. For a definitive version of this work, please refer to the published source. Please note that access to the published version might require a subscription.

Chalmers Publication Library (CPL) offers the possibility of retrieving research publications produced at Chalmers University of Technology. It covers all types of publications: articles, dissertations, licentiate theses, masters theses, conference papers, reports etc. Since 2006 it is the official tool for Chalmers official publication statistics. To ensure that Chalmers research results are disseminated as widely as possible, an Open Access Policy has been adopted.

The CPL service is administrated and maintained by Chalmers Library. 


\title{
Nonlinear 3-D Microwave Imaging for Breast-Cancer Screening: Log, Phase and Log-Phase Formulation
}

\author{
Peter D. Jensen*, Tonny Rubæk ${ }^{\dagger}$, Johan J. Mohr* and Vitaliy Zhurbenko* \\ pdj@elektro.dtu.dk, rubaek@chalmers.se, jm@elektro.dtu.dk, vz@elektro.dtu.dk
}

*Department of Electrical Engineering, Electromagnetic Systems, Technical University of Denmark, Ørsteds Plads, Building 348, DK-2800 Kgs. Lyngby, Denmark.

$\dagger$ Department of Signals and Systems, Biomedical Engineering, Chalmers University of Technology, SE-41296 Gothenburg, Sweden.

\begin{abstract}
The imaging algorithm used in the 3-D microwave imaging system for breast cancer screening, currently being developed at the Technical University of Denmark, is based on an iterative Newton-type algorithm. In this algorithm, the distribution of the electromagnetic constitutive parameters is updated in each iteration based on a comparison between the measured signals and the signals computed by a forward solver for the current parameter distribution. A key feature of the algorithm is the use of the log-phase formulation in which the measured complex signals are represented by the logarithm of their amplitudes and their unwrapped phases. In this paper, simplifications of the log-phase formulation are proposed, namely the log formulation, in which only the logarithm of the amplitudes are used, and the phase formulation, in which only the unwrapped phases are used. These formulations allow for the use of simpler tomographic systems in which only the amplitudes or the phases of the signals are measured. The performance of the algorithms using the two proposed formulations is investigated by reconstructing images of data collected with the above-mentioned imaging system, and the resulting images are compared with those obtained using the log-phase formulation.
\end{abstract}

\section{INTRODUCTION}

Microwave imaging for breast-cancer screening has attracted the attention of an increasing number of research groups during the last decade, and is seen as a possible supplement to the widely used X-ray mammography.

Today's trend in microwave imaging for breast-cancer detection goes towards 3-D measurement setups to avoid the inherent errors stemming from applying 2-D algorithms to what indeed is a 3-D problem [1]. Two different approaches are currently being pursued for microwave imaging of the breast: One is the ultrawideband radar-based approach, in which the images are constructed by determining the pointof-origin of reflected pulses; the other is the tomographic or inverse-scattering approach in which the images are created by solving an inverse-scattering problem based on Maxwell's equations. This approach results in a reconstruction of the distribution of the constitutive electromagnetic properties, i.e., permittivity and conductivity, of the breast.

The prototype system, currently being developed at the Technical University of Denmark (DTU), belongs to the latter group. It is a 3-D imaging system that uses an iterative Newton-type algorithm for solving the inverse problem. In this algorithm, the distribution of electromagnetic constitutive parameters is updated in each iteration based on a comparison between the measured signals and the signals computed by a forward solver for the current parameter distribution.

It has earlier been shown that the so-called log-phase formulation, in which the measured signals are represented by the logarithm of their amplitudes and their unwrapped phases, significantly improves the performance of the reconstruction algorithm compared to the complex formulation [2]. In this paper, simplifications of the log-phase formulation are proposed. These simplified formulations consist of two different algorithms using only the amplitude or only the phase, respectively, of the signals.

The use of such formulations would enable the use of simpler tomographic systems that measure only either the amplitude or the phase of the signals at the terminals of the antennas. For an amplitude-only system, the measurements are simplified since there is no longer need for coherent measurements. This also allows for a complete decoupling between the transmitting and receiving units, since the individual transmitter and receiver units no longer need to have a common feed to their local oscillators (LO). This, in turn, can eliminate signals leaking from the transmitter through the common LO channels to the receiver. A problem which can seriously affect the performance of tomographic systems in which signal levels more than $120 \mathrm{~dB}$ below the transmitted signal are often observed on the receiving channels [3].

In a phase-only measurement system, on the other hand, the requirements to the $\mathrm{AD}$ converter in the system can be relaxed and so can the requirements to the overall dynamic performance, which is most often needed in these systems to handle the large changes in amplitudes, observed by the receiving antennas depending on their relative position to the transmitting antenna.

Hence, it is of great interest to investigate whether or not satisfactory results can be obtained using either amplitude-only or phase-only measurements, thereby relaxing the requirements 
to the hardware of the tomography system.

\section{IMAGING SYSTEM}

The DTU system is designed to be operated at a single frequency in the range from $300 \mathrm{MHz}$ to $3 \mathrm{GHz}$. The system consists of 32 monopole antennas submerged in a glycerinwater coupling liquid. Each of the antennas are equipped with its own transceiver module. The antennas are positioned in a cylindrical setup with 4 rows. Each row consists of 8 antennas equally spaced by $45^{\circ}$ on a circle with radius $8 \mathrm{~cm}$. The distance between the rows is $2.5 \mathrm{~cm}$, and the individual rows are rotated $22.5^{\circ}$ with respect to the rows above and below [3].

The antenna system is positioned in a cylindrical tank with a radius of $20 \mathrm{~cm}$. The lossy coupling liquid assures that reflections from the side, bottom and top of tank are attenuated so the influence of reflections becomes negligible. During a measurement sequence, each antenna in turn transmits while the response is measured on the 31 other antennas, yielding 992 measurement of the amplitude and phase. Due to reciprocity the measurements are pairwise redundant.

\section{IMAGING ALgORITHM}

The distribution of electromagnetic constitutive parameters in the imaging domain can be represented by the squared complex wave number. Using the time notation $e^{j \omega t}$, the squared complex wave number in the domain is given by

$$
k^{2}(\boldsymbol{r})=\omega^{2} \mu_{0} \epsilon(\boldsymbol{r})-j \omega \mu_{0} \sigma(\boldsymbol{r}),
$$

where $\mathbf{r}$ is the position vector, $\omega$ is the angular frequency, $\mu_{0}$ is the free-space permeability, $\epsilon$ is the permittivity, and $\sigma$ is the conductivity. The domain is discretized into $N_{\text {cells }}$ cubic cells, and the constitutive parameters are assumed to be constant within each cell. Then, it holds that

$$
k^{2}(\boldsymbol{r})=k_{l}^{2} \quad \text { for } \quad \boldsymbol{r} \in V_{l},
$$

where $V_{l}$ is the volume covered by cell number $l=$ $1,2, \ldots, N_{\text {cells }}$.

The distribution of the squared complex wave numbers in the imaging domain is determined by solving the nonlinear minimization problem, given by

$$
\begin{aligned}
\boldsymbol{k}^{2} & =\operatorname{argmin}\left\{\left\|\boldsymbol{S}^{\text {meas }}-\boldsymbol{S}^{\text {calc }}\left(\boldsymbol{k}^{2}\right)\right\|_{2}^{2}\right\} \\
& =\operatorname{argmin}\left\{\left\|\boldsymbol{S}^{\text {res }}\left(\boldsymbol{k}^{2}\right)\right\|_{2}^{2}\right\} \text { subj. to regularization }
\end{aligned}
$$

using an iterative Newton algorithm. In this expression, the $2 N_{\text {cells }}$-element vector $\boldsymbol{k}^{2}$ holds the distribution of real and imaginary parts of the squared complex wave numbers $k_{l}^{2}$. The vectors $\boldsymbol{S}^{\text {calc }}\left(\boldsymbol{k}^{2}\right)$ and $\boldsymbol{S}^{\text {meas }}$ holds the calculated signals for a given distribution of the squared wave numbers and the measured signals, respectively. $\boldsymbol{S}^{\text {res }}$ is the residual vector.

Since the problem is both under determined and ill-posed, regularization must be applied when solving the problem. In this paper, the Newton algorithm outlined in [4] is applied for solving the inversion problem. And the regularization is obtained by solving for the update problem with a limited number of iterations (here 6) in the CGLS algorithm, corresponding to applying over-regularization [5, Sec. 6.3].

\section{Log, Phase And Log-Phase Formulation}

It has earlier been shown that the log-phase formulation has advantages compared to the complex formulation [2]. The complex and log-phase formulations differ in how the data is represented in the vectors $\boldsymbol{S}^{\text {meas }}$ and $\boldsymbol{S}^{\text {calc }}\left(\boldsymbol{k}^{2}\right)$. In both formulations, each combination of transmitting antenna $t$ and receiving antenna $r$ gives two elements in each of the vectors $S^{\text {meas }}$ and $\boldsymbol{S}^{\text {calc }}\left(\boldsymbol{k}^{2}\right)$. In the complex formulation, the elements are given by

$$
S_{\Re}^{t, r}=\operatorname{Re}\left\{S_{\mathrm{obj}}^{t, r}-S_{\mathrm{empty}}^{t, r}\right\}
$$

and

$$
S_{\Im}^{t, r}=\operatorname{Im}\left\{S_{\mathrm{obj}}^{t, r}-S_{\mathrm{empty}}^{t, r}\right\},
$$

where $S_{\text {obj }}^{t, r}$ and $S_{\text {empty }}^{t, r}$ denotes measurements with and without an object present in the imaging system, respectively.

In the log-phase formulation, the vectors are based on the logarithm of the signals

$$
\log S^{t, r}=\log \left|S^{t, r}\right|+j \angle S^{t, r},
$$

wherein $\angle S^{t, r}$ is the unwrapped phase of the signal. The $2 N_{\text {meas }}$ elements of the vectors $S^{\text {meas }}$ and $S^{\text {calc }}$ may now be determined as

$$
S_{\log }^{t, r}=\log \left|S_{\mathrm{obj}}^{t, r}\right|-\log \left|S_{\text {empty }}^{t, r}\right|
$$

and

$$
S_{\text {phase }}^{t, r}=\angle S_{\text {obj }}^{t, r}-\angle S_{\text {empty }}^{t, r} .
$$

In the log formulation proposed in this paper, it is assumed that only the amplitudes of the signals are given. The elements in the vectors $\boldsymbol{S}^{\text {meas }}$ and $\boldsymbol{S}^{\text {calc }}\left(\boldsymbol{k}^{2}\right)$ are then determined using (7) and the number of elements in the vectors is half of the corresponding number when using the log-phase formulation, i.e., $N_{\text {meas }}$.

Similarly, in the proposed phase formulation, it is assumed that only the phases of the signals are given. The elements in the vectors $\boldsymbol{S}^{\text {meas }}$ and $\boldsymbol{S}^{\text {calc }}\left(\boldsymbol{k}^{2}\right)$ are thus determined using (8) and the number of elements in each of the vectors is $N_{\text {meas }}$.

In this paper, the phase-only and amplitude-only data sets are created from a measurement containing both amplitude and phase but the data sets could instead be obtained from systems which only measure one or the other.

\section{RESUlTS AND DisCUSSION}

To illustrate the performance of the proposed log and phase algorithms compared with that of the log-phase algorithm, the results from the three imaging setups shown in Fig. 1 will be discussed. Configuration 1 and 2 consist of thin plastic spheres with a diameter of $4 \mathrm{~cm}$ that are filled with tap water and positioned in the coupling liquid of the imaging system. The centers of the spheres are placed in the $x y$ plane with $z=-4 \mathrm{~cm}$, i.e., aligned with the third row of antennas, counting from the top. For configuration 1, the center of the sphere is located at $(x, y)=(2.2,-2.7) \mathrm{cm}$. In configuration 2 , the spheres form a equilateral triangle with a side length of $6 \mathrm{~cm}$, with the sphere in the top-right corner 


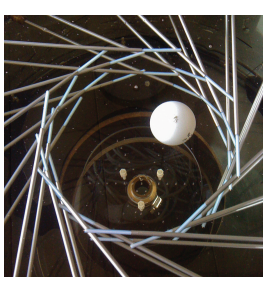

(a) Configuration 1 .

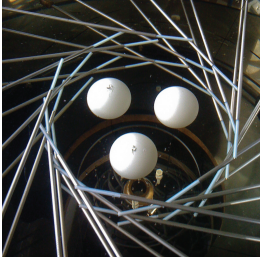

(b) Configuration 2

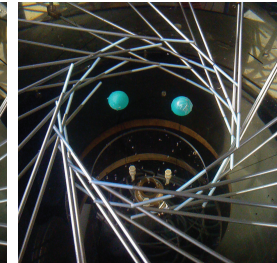

(c) Configuration 3
Fig. 1. Water filled plastic spheres submerged in coupling liquid. Results for configuration 1, 2 and 3 are presented in Fig. 2, Fig. 3 and Fig. 4, respectively.

being at the same position as the sphere in configuration 1 . Each floating sphere is held in place with a piece of thread fixed to the bottom of the tank.

Configuration 3, shown in Fig. 1(c) consists of two plastic spheres with a diameter of $2 \mathrm{~cm}$ that are filled with tap water. These are less ideal test objects than the larger spheres used in the first two configurations because more volume is taken up by the plastic (the spheres are approximately $1 \mathrm{~mm}$ thick, implying that only $73 \%$ of the total volume is water). The spheres are positioned with their centers at the same positions as the two upper spheres in configuration 2.

The used imaging domain is cylindrically shaped with a diameter of $14 \mathrm{~cm}$ and a height of $8.5 \mathrm{~cm}$ with the top positioned at $z=0 \mathrm{~cm}$. It is divided into 10421 cubic cells with a side length of $0.5 \mathrm{~cm}$.

Fig. 2 shows images of the reconstructed relative permittivity and conductivity for configuration 1 at $1 \mathrm{GHz}$. The images are slices in the $x y$-plane at $z=-4.00 \mathrm{~cm}$. The relative permittivity of tap water and the coupling liquid is approximately 80 and 10.5 , respectively. The conductivity values are approximately $0.25 \mathrm{~S} / \mathrm{m}$ for tap water and $0.7 \mathrm{~S} / \mathrm{m}$ for the coupling liquid.

Figs. 2(a) and (b) show log-phase formulation results. The sphere stands out clearly in the image of the permittivity. It is circularly shaped and it is positioned as expected. The reconstructed relative permittivity has a maximum of $\epsilon_{r} \approx 55$. As expected, the conductivity of the object is lower than the surrounding liquid in the image, but the sphere is not as clearly defined as in the permittivity image and artifacts can be seen around the sphere. Overall, though, the images obtained using log-phase formulation are in good agreement with the actual values.

Figs. 2(c) and (d) show log formulation results. Fig. 2(c) looks very similar to Fig. 2(a), with a slightly lower maximum permittivity of $\epsilon_{r} \approx 51$. As with the log-phase formulation, the reconstructed conductivity in Fig. 2(d) is dominated by artifacts and the sphere is not clearly defined.

Fig.2(e) and (f) show phase formulation results. Again, the image of the permittivity is good, and the sphere is clearly defined, but with an slightly lower maximum value of $\epsilon_{r} \approx 50$. As with the two previous examples, the sphere is not clearly defined in the conductivity image in Fig. 2(f).

Fig. 3 shows the reconstructed images for configuration 2 using the different formulations. Again, the spheres clearly

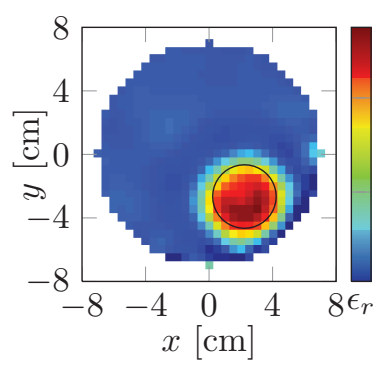

(a) Log-phase formulation.

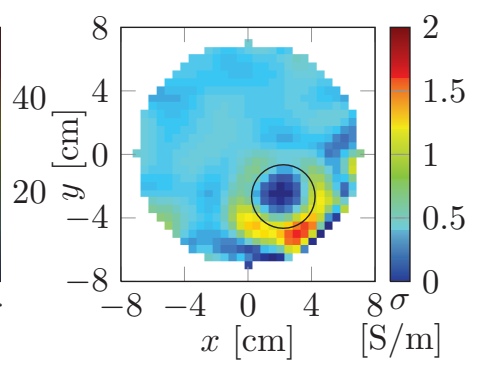

(b) Log-phase formulation.

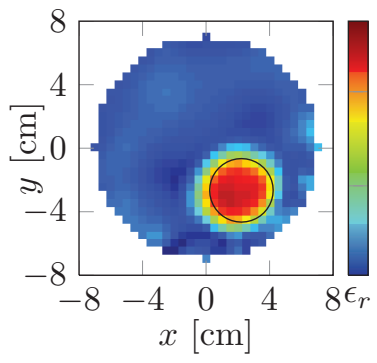

(c) Log formulation.

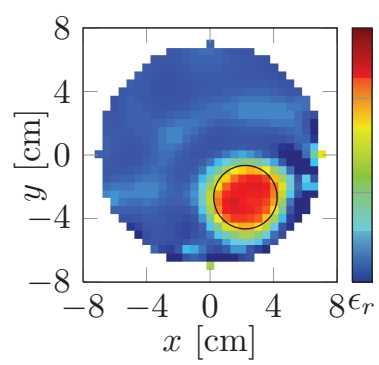

(e) Phase formulation.

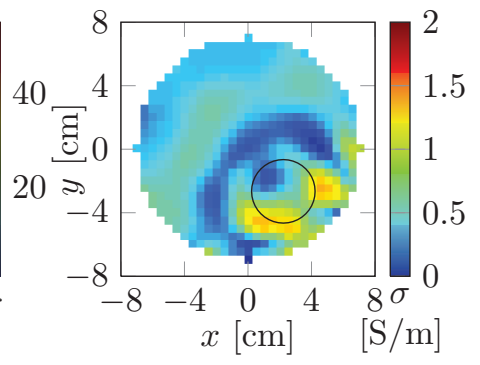

(d) Log formulation

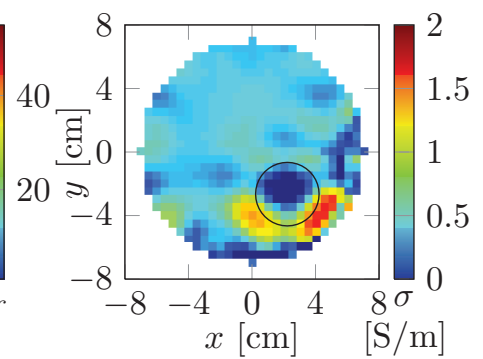

(f) Phase formulation.

Fig. 2. Configuration 1: Images of the reconstructed relative permittivity, $\epsilon_{r}=\epsilon / \epsilon_{0}$, and conductivity, $\sigma$, in the $x y$-plane at $z=-4.00 \mathrm{~cm}$, at $1 \mathrm{GHz}$, after 20 Newton iterations using the three different formulations. The black circles indicate the actual position.

stand out in the permittivity images. The maximum values of the reconstructed permittivity for the log-phase, log and phase formulation are 63,58 and 53 , respectively. The image of the permittivity obtained using phase formulation looks notably smeared compared to the other two permittivity images.

As was the case with configuration 1, the spheres are not well defined in the conductivity images which are dominated by artifacts.

Fig. 4 shows the reconstruction of configuration 3 that consist of 2 smaller spheres. The reconstructions are done at the frequency $1.5 \mathrm{GHz}$. At this frequency, the relative permittivity is approximately 80 for tap water and 7.8 for the coupling liquid. The corresponding values for the conductivity is approximately $0.5 \mathrm{~S} / \mathrm{m}$ and $0.8 \mathrm{~S} / \mathrm{m}$. The small spheres clearly stand out in the permittivity images. The small spheres are also seen in the conductivity images using log-phase and phase formulation. The spheres are not visible in the conductivity image obtained with the log formulation.

In addition to the examples shown here, a number of other 


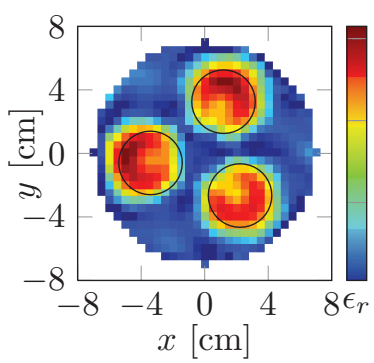

(a) Log-phase formulation.

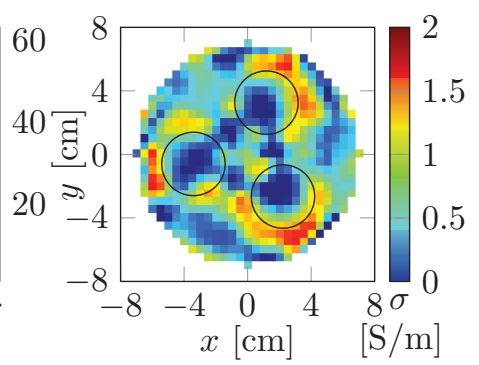

(b) Log-phase formulation.

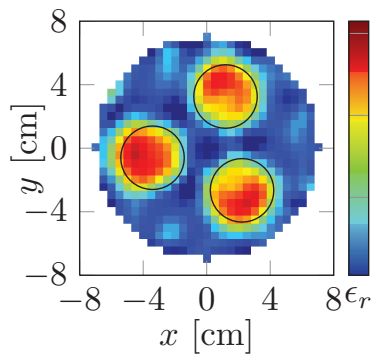

(c) Log formulation.

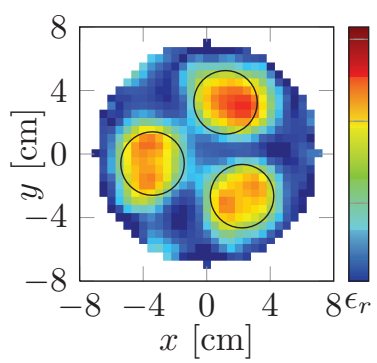

(e) Phase formulation.

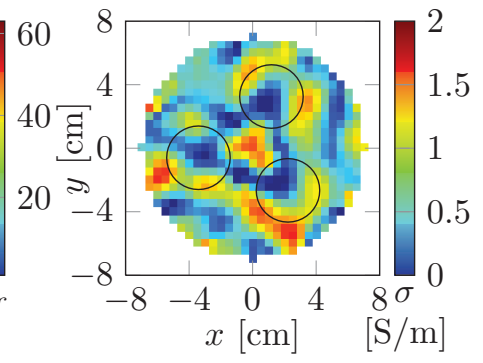

(d) Log formulation.

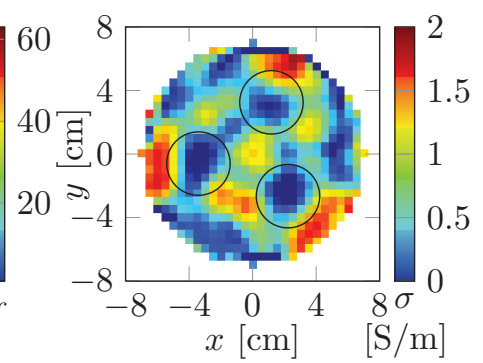

(f) Phase formulation.
Fig. 3. Configuration 2: Images of the reconstructed relative permittivity, $\epsilon_{r}=\epsilon / \epsilon_{0}$, and conductivity, $\sigma$, in the $x y$-plane at $z=-4.00 \mathrm{~cm}$, at $1 \mathrm{GHz}$ after 20 Newton iterations using the three different formulations. The black circles indicate the actual position.

imaging experiments have been carried out. These all show similar results to the examples shown here, i.e., that the phase formulation yields results that are comparable to those of the log-phase formulation, while the log formulation yields slightly worse results. Especially for the conductivity images.

\section{CONCLUSION}

Two simplifications of the log-phase formulation have been proposed, one being the log formulation in which only the amplitude measurements are used, the other being the phase formulation in which only the phase measurements are used. The formulations have been tested with data collected with a 3-D microwave imaging system, and the images compared with those obtained using the log-phase formulation.

The results show that a simplified imaging system, either measuring the amplitude or the phase of the signals, gives satisfactory results that are in many cases comparable to the results obtained with a full measurements of both amplitude and phase. However, the phase-only system seems to yield better images compared to the amplitude-only system.

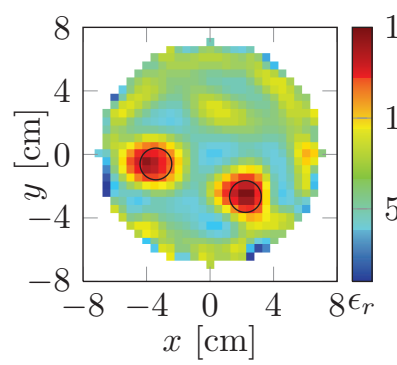

(a) Log-phase formulation.

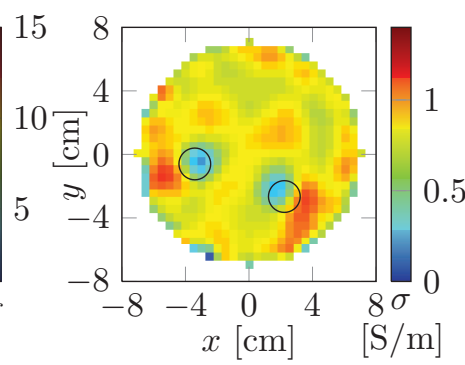

(b) Log-phase formulation.

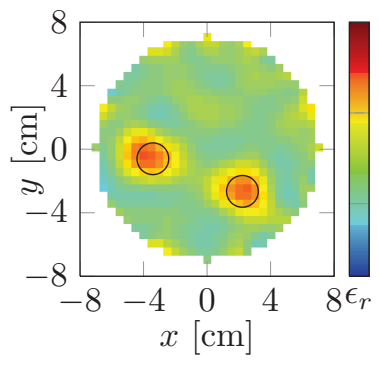

(c) Log formulation.

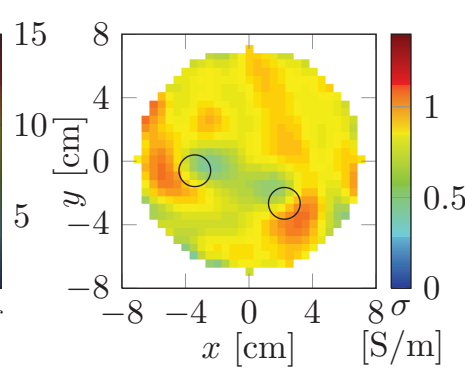

(d) Log formulation.

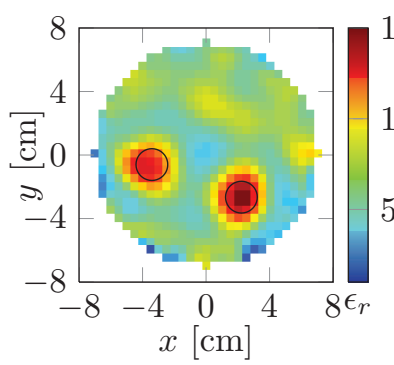

(e) Phase formulation.

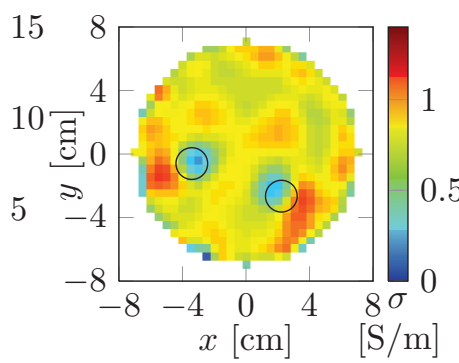

(f) Phase formulation.
Fig. 4. Configuration 3: Images of the reconstructed relative permittivity, $\epsilon_{r}=\epsilon / \epsilon_{0}$, and conductivity, $\sigma$, in the $x y$-plane at $z=-4.00 \mathrm{~cm}$, at 1.5 $\mathrm{GHz}$, after 20 Newton iterations using the three different formulations. The black circles indicate the actual position.

\section{ACKNOWLEDGMENTS}

The work presented in this paper is, in part, funded by the Villum Kann Rasmussen Foundation.

\section{REFERENCES}

[1] P. Meaney, K. Paulsen, S. Geimer, S. Haider, and M. Fanning, "Quantification of 3-d field effects during 2-d microwave imaging," IEEE Transaction on Biomedical Engineering, vol. 49, no. 7, pp. 708-720, Jan 2002.

[2] P. Meaney, K. Paulsen, B. Pogue, and M. Miga, "Microwave image reconstruction utilizing log-magnitude and unwrapped phase to improve high-contrast object recovery," IEEE Transactions on Medical Imaging, vol. 20, no. 2, pp. 104-116, 2001.

[3] V. Zhurbenko, T. Rubæk, V. Krozer, and P. Meincke, "Design and realisation of a microwave three-dimensional imaging system with application to breast-cancer detection," IET Microwaves, Antennas \& Propagation, vol. 4, no. 12, pp. $2200-2211,2010$.

[4] T. Rubæk, O. Kim, and P. Meincke, "Computational validation of a 3-d microwave imaging system for breast-cancer screening," IEEE Transactions on Antennas and Propagation, vol. 57, no. 7, pp. 2105-2115, 2009.

[5] P. C. Hansen, Rank-Deficient and Discrete Ill-Posed Problems: Numerical Aspects of Linear Inversion, ser. Monographs on Mathematical Modeling and Computation. SIAM, 1998. 